\title{
Distribución de las tensiones en el sistema pared-mortero-bizcocho-esmalte
}

Prof. Dr. A. DIETzEL, y

Prof. Dr. H. J. OEL

Ceramica Informazione, $n^{\circ} 52$, enero 1971, págs. 16-24

Cuando en los azulejos (placas de revestimiento) aparecen tensiones después de su colocación, la consecuencia inmediata son dos inconvenientes desagradables: 1) el eventual despegue del azulejo después de un período de tiempo más o menos corto, por desplazamiento de una pieza sencilla o de las filas enteras; 2) la sucesiva aparición de fisuras capilares en el esmalte.

En ambos casos se atribuye la culpa a la expansión del bizcocho cerámico, por efecto de la humedad, que tiene lugar después de la colocación de los azulejos. Sin embargo, algunas observaciones prácticas nos sugieren que la expansión por humedad no es la única causa. En la realidad, por regla general, la loseta se pega muy bien al mortero y a la pared; y dicho mortero por tener el mismo coeficiente de dilatación que el bizcocho, da lugar a poquísimos problemas, mejor dicho no es corriente que aparezcan totalmente incluso en largos períodos de tiempo.

El objeto de esta investigación es aclarar estos conceptos contrastantes y establecer, por una parte, si el tipo de arena tiene influencia sobre el mortero y en qué medida, y, por otra, ver de qué modo distintas cantidades de agua embebida ejercen influencia sobre el azulejo.

\section{PARTE PRIMERA}

\section{Plaquetas y tensiones relativas en el sistema bizcocho-esmalte}

\section{A. DIETZEL.}

Para estos experimentos se contó con diversos tipos de azulejos de la producción corriente de dos importantes fábricas, siendo cuatro los tipos de bizcochos y seis los tipos de esmalte. Sólo hemos tenido en consideración un tipo de bizcocho y dos esmaltes diferentes, siendo estos un barniz de plomo y un barniz de circonio.

Tres de los cuatro bizcochos tenían características un tanto parecidas. Las diferencias no eran mayores que las existentes entre azulejo y azulejo de una misma fábrica, e, incluso, que las que hay en un mismo azulejo. Unicamente el cuarto bizcocho mostraba un notable contenido en cristobalita, a consecuencia de la cual cabía esperar una menor predisposición a la formación de huecos capilares. Sin embargo, este bizcocho no se ha tomado en consideración, puesto que este problema se deberá examinar adecuadamente. 
En lo que se refiere a los esmaltes, los que eran a base de plomo se comportaron del mismo modo entre sí, y, lo mismo sucedió con los barnices a base de circonio. Entre ambos tipos de esmalte se distinguen máximos para su reactividad, como se demostrará más adelante. Además de las piezas de bizcocho esmaltado también se tuvieron a disposición piezas de bizcocho sin esmaltar, haciéndose numerosas pruebas, de las cuales solamente hablaremos de las más características e interesantes.

En la tabla 1 se indican los contenidos en $\mathrm{CaO}$ y en $\mathrm{Na}_{2} \mathrm{O}$ del bizcocho, ambos determinados por análisis químico, $\mathrm{y}$, también, la absorción de agua.

T A B L A I

\begin{tabular}{lccc}
\hline & $\begin{array}{c}\mathrm{CaO} \\
(\%)\end{array}$ & $\begin{array}{c}\mathrm{Na}_{2} \mathrm{O} \\
(\%)\end{array}$ & $\begin{array}{c}\text { Absorción de agua } \\
(\%)\end{array}$ \\
\hline Bizcocho & 2,23 & 1,87 & 15,4 \\
\hline
\end{tabular}

En la figura 1 se muestra la curva dilatométrica del bizcocho normal, que se encuentra situada entre la curva del bizcocho cristobalítico y las curvas de las dos clases de barnices.

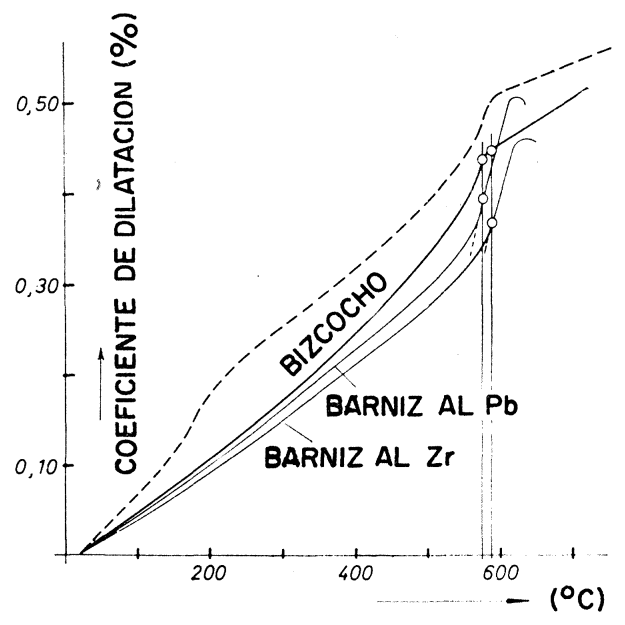

Fig. 1.-Dilataciones térmicas del bizcocho normal, del barniz de plomo y del barniz de circonio. La línea de trazos corresponde al bizcocho cristobalítico, trazados como término de comparación.

La dilatación térmica del barniz de plomo entre $23^{\circ} \mathrm{C}$ y su temperatura de "templado" ET (1) $=576^{\circ} \mathrm{C}$, era del $0,397 \%$ y la del barniz de circonio, para una $\mathrm{ET}=588^{\circ} \mathrm{C}$, era del $0,367 \%$.

La dilatación del bizcocho entre estas mismas temperaturas fue de:

- entre 23 y $576^{\circ} \mathrm{C}$

$0,440 \%$

- entre 23 y $588^{\circ} \mathrm{C}$ $0,448 \%$;

es decir, mayor que las de los barnices.

Las diferencias de las dilataciones fueron:

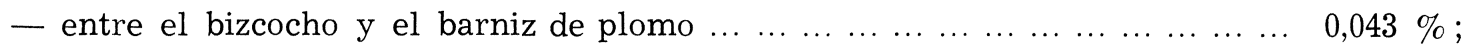

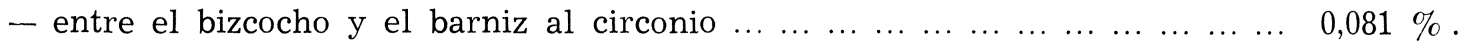


Por consiguiente, en este caso es de esperar que ambos barnices se encuentren bajo solicitaciones de compresión.

Sobre diversos azulejos tratados y, en barritas de barniz, se determinó el módulo E. Como puede verse en la tabla II este valor disminuye en los azulejos saturados en agua y notablemente después de una larga permanencia en la misma.

T A B L A II

Módulo $E\left(\mathrm{kp} / \mathrm{mm}^{2}\right)$ para materiales cocidos a $1.000^{\circ} \mathrm{C}$

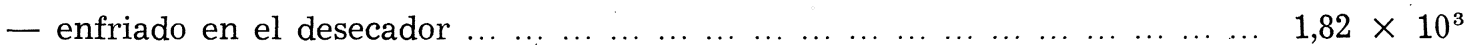

- saturado en agua $\ldots$
$\ldots$

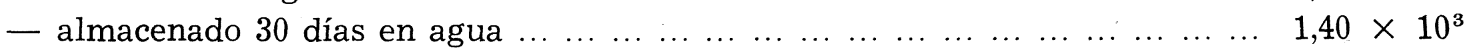

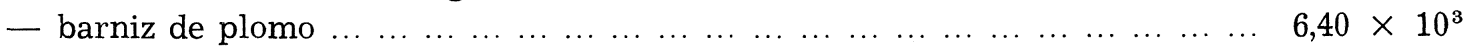

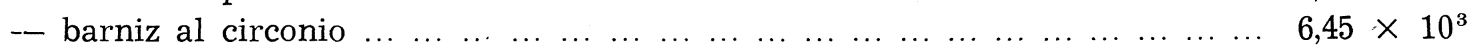

Interesaba, ante todo, determinar la dilatación por humedad. Esto se determinó de dos maneras: una de ellas dejando sumergidas durante más de 50 días pequeñas barras de $5 \times 10 \times 100 \mathrm{~mm}$, cortadas de una placa no esmaltada; la otra colocando plaquetas esmaltadas y otras sin esmaltar en la autoclave a 2 atmósferas y 13 horas y a 10,5 atmósferas y 1 hora, respectivamente. Los resultados obtenidos pueden verse en las figuras 2 y 3 .

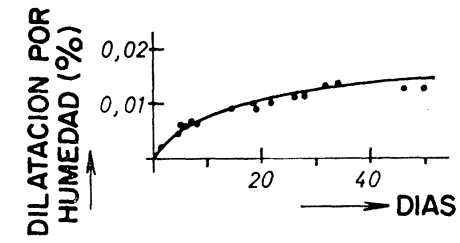

Fig. 2.-Dilatación por humedad del bizcocho sin esmaltar, por inmersión en agua.

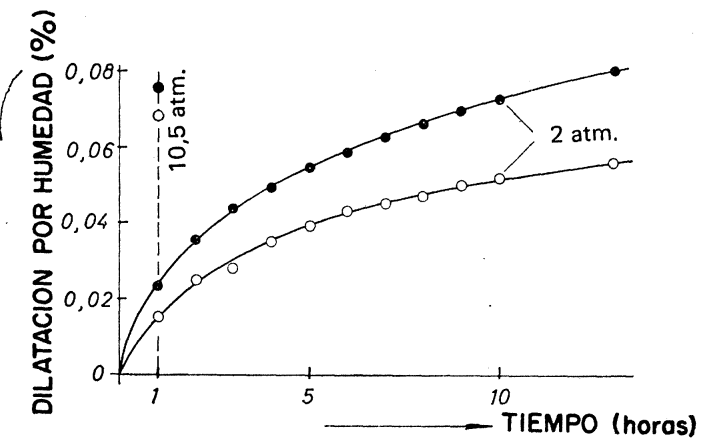

Fig. 3.-Dilatación por humedad del bizcocho con $\circ$ o sin • esmalte, tratado en autoclave a 2 atm, (en particular después de 1 hora a $10,5 \mathrm{~atm}$ ).

Ya se ha publicado en diferentes trabajos y se ha podido comprobar en la práctica cuál es la temperatura en la que se anula más o menos completamente la dilatación por humedad. Generalmente esto sucede entre $400^{\circ}$ y $500^{\circ} \mathrm{C}(2)$.

Sin embargo, hemos llegado a la conclusión, por medidas hechas sobre diversos bizcochos, que es necesaria una temperatura próxima a la original de cocción, es decir, de unos $1.000^{\circ} \mathrm{C}$, como puede verse en la figura 4 . En vista de que las plaquetas de que se disponía para los experimentos eran de precedentes desconocidos y, además, habían estado almacenadas durante cierto tiempo, hubo de tenerse en cuenta el hecho de que tenían que 
haber absorbido humedad. En consecuencia, se las sometió a una nueva cocción durante una hora a $1.000^{\circ} \mathrm{C}$ y se las dejó enfriar lentamente. Por último se conservaron así en los desecadores.

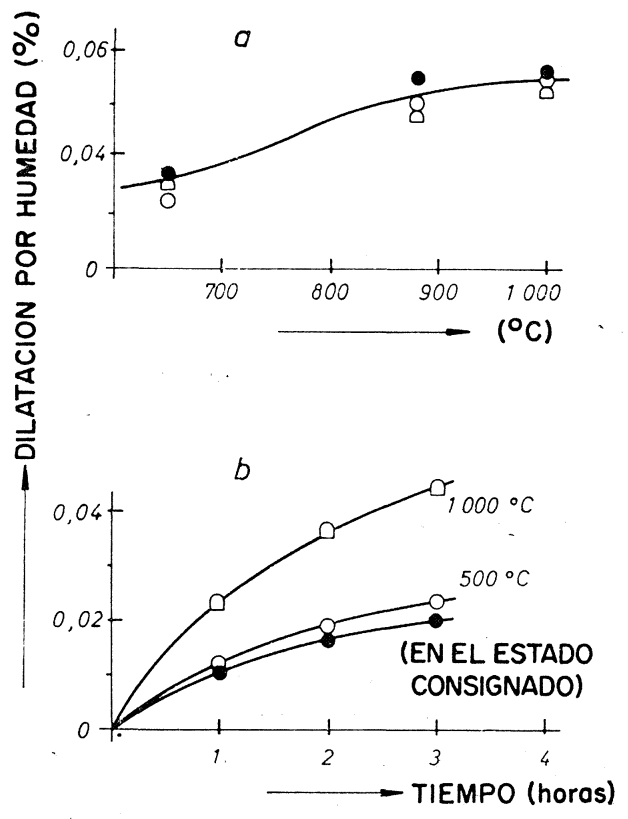

Fig. 4.-Dilatación del bizcocho por humedad con diversas temperaturas de recocido: a) Dilatación por tratamiento en autoclave a 2 atm, durante 12 horas, en relación con la temperatura de cocción previa. Azulejo esmaltado tal como viene del proveedor. b) Dilatación de una placa sin esmaltar, tratada en autoclave a 2 atm, en función del tiempo, tal como viene del proveedor $y$, en particular, recocida.

\section{Discusión de los resultados}

De la figura 2 se puede apreciar que a temperatura ambiente, al cabo de 50 días, la placa sin esmaltar presentaba una dilatación por humedad del $0,015 \%$, mientras que a los 10 días era inferior al $0,010 \%$.

Estos valores son muy inferiores a los que resultan de la diferencia de las dilataciones térmicas existentes entre el bizcocho y los barnices.

Sin embargo, se observó que en aquellas placas en las que se había aplicado el barniz de plomo aparecieron, al cabo de 7 días, o a más tardar 14 días, las primeras fisuras capilares en el barniz. También es un hecho bien sabido por la experiencia, que el barniz de plomo, a diferencia con el de circonio, tiene una particular tendencia a la formación de cavidades. El examen Steger no ha aclarado nada sobre este particular: Esto ha sido apreciado asimismo en el barniz de plomo bajo solicitación de compresión. El efecto es todavía más patente si se observa también el alargamiento del esmalte debido a la formación del surco.

Se ha calculado que es del $0,067 \%$ con surcos de $1 \mathrm{~mm}$ de espesor y $100 \mathrm{~mm}$ de longitud, para el barniz de plomo y del 0,084 \%, para el barniz de circonio.

De lo que se deduce que se debe esperar en el barniz de plomo una dilatación del 0,043\% (neutralización de la compresión debida al bizcocho) + 0,067\% (alargamiento de rotura) $=0,110 \%$, hasta que se produce el cuarteamiento. En este cálculo no interviene la dilatación por humedad, aún después del riguroso tratamiento en autoclave, ya que aquélla, según puede verse en la figura 3, en el caso del bizcocho no esmaltado mantenido durante 1 hora a 10,5 atm, es de tan sólo un $0,075 \%$, y de un 0,055 \% para la plaque- 
ta esmaltada mantenida durante 12 horas a 2 atm. Para las placas sin esmaltar mantenidas durante 12 horas a $2 \mathrm{~atm}$, fue del $0,080 \%$. Por tanto, la dilatación por humedad no es suficiente para que se produzca este ligero cuarteo en los barnices de plomo, y por esta razón deben forzosamente entrar en juego otros factores.

\section{Investigaciones con microscopio}

Mediante una investigación realizada con el microscopio, sobre láminas cortadas transversalmente de las placas esmaltadas, se han podido comprobar determinados efectos. Mientras que el esmalte al circonio, que se vuelve opaco, presenta una pequeña zona reactiva con el soporte de bizcocho (fig. 5 a), en el esmalte de plomo esta zona de reacción abarca más de un tercio del espesor del esmalte (fig. 5 b).

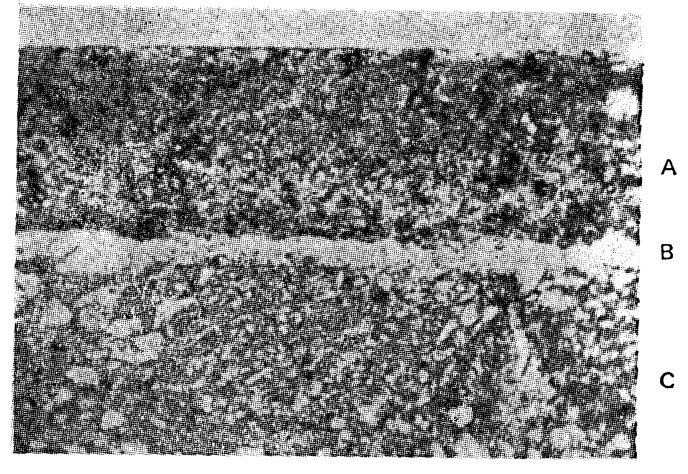

Fig. 5a.-Lámina transversal del bizcocho con esmalte al circonio. Pequeña zona de reacción $B$ (clara) entre el esmalte A (encima) y el bizcocho C (debajo). (185 x).

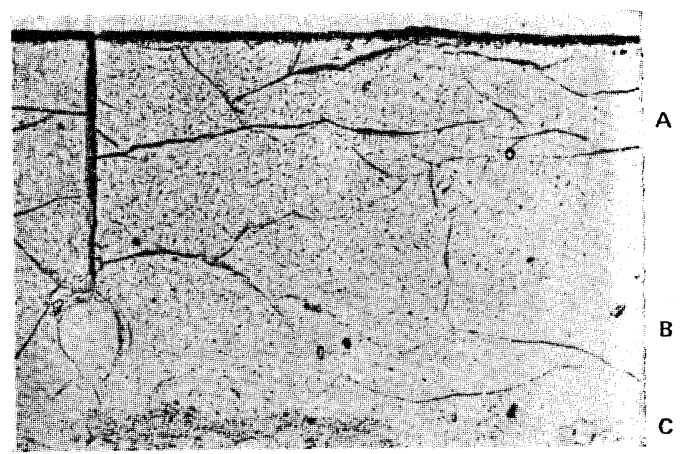

Fig. 5b.-Lámina transversal de una placa con barniz de plomo. A, barniz de plomo, $B$ capa intermedia, $C$ bizcocho $(185 \mathrm{x})$. La fisura vertical de la izquierda sólo se desarrolla en la capa $A$ que está en tensión.

Se observó asimismo que el índice de refracción del estrato vitrificado disminuye considerablemente en dirección al bizcocho (fig. 6).

Lo cual significa que se ha combinado una considerable cantidad del esmalte, a base de barniz de plomo, con el bizcocho. Es bien conocido el ataque que producen los vidrios a ios materiales refractarios, (3) y recientemente se sabe lo que sucede entre la porcelana (4) y el esmalte que, en los estratos intermedios, no es una simple mezcla del material cerámico con el vidrio o con el esmalte, para lo cual necesitamos estudiar los procesos selectivos de difusión.

Esto es válido dentro de ciertos límites, así como en el caso que se está ahora examinando. Efectivamente, el contenido en álcalis del barniz de plomo era relativamente bajo. Si se tratase de una difusión en la dirección del bizcocho, deberá aumentar la refracción de la luz en dicho barniz de plomo, hecho que a veces no se observó. En el bizcocho, además, la parte de componente vítreo era pequeña, debido a que la temperatura de cocción fue muy moderada (cosa totalmente diferente en la chamota de la porcelana).

Por consiguiente, la deficiencia no tiene gran importancia si, como ya se ha advertido, se trata de un derretimiento del bizcocho en el esmalte. A tales efectos, de los fundidos de esmalte y diversas fracciones de bizcocho se prepararon piezas de las que se determinó la refracción (fig. 7). 
De esta manera se ha podido determinar, por aproximaciones, midiendo la refracción de la luz, la composición del estrato intermedio, según los diversos grados de absorción del bizcocho (fig. 6).

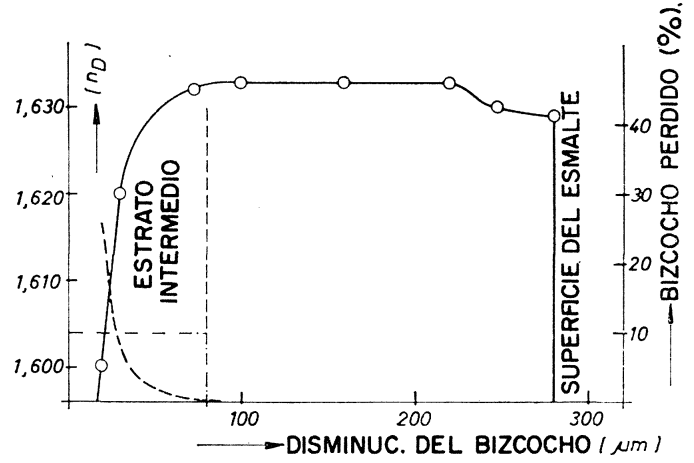

Fig. 6.-Valores de las refracciones de la luz a lo largo de la sección transversal de un esmalte. Cantidad de bizcocho perdido en el estrato intermedio calculada con la ayuda de la figura 7 (curva de trazos y escala de la derecha).

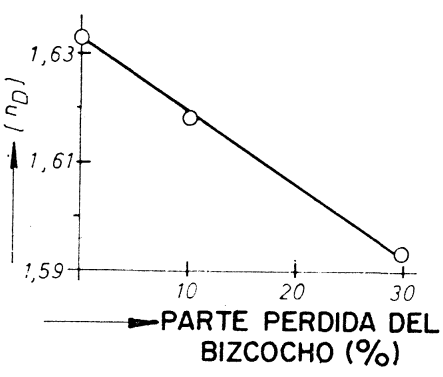

Fig. 7.-Refracción del barniz de plomo con $y$ sin pérdida de bizcocho.

Normalmente $1 / 3$ del espesor del barniz está alterado por la interfase con el bizcocho. (Por otra parte, la refracción de la luz se produjo un poco por debajo de la superficie, evidentemente a causa de la evaporación del $\mathrm{PbO}$ ). Como valor medio de la interfase del bizcocho en el esmalte, para un espesor de $80 \mu \mathrm{m}$, se puede considerar un $10 \%$, aproximadamente.

En un esmalte de la presente composición (10\% de bizcocho $+90 \%$ de esmalte) se ha determinado el comportamiento por dilatación. La temperatura de "templado" (ET) fue de $605^{\circ} \mathrm{C}$. La dilatación térmica entre $20^{\circ} \mathrm{C}$ y ET $\left(605^{\circ} \mathrm{C}\right)$ fue del $0,378 \%$, pero entre $20^{\circ} \mathrm{C}$ y ET $\left(576^{\circ} \mathrm{C}\right)$ del barniz de la parte superior no influenciado, fue del 0,309\%.

Por eso la interfase, en el sistema bizcocho-barniz, tiene una dilatación térmica mínima; y respecto a este estrato intermedio, la capa de barniz de la parte superior debe encontrarse bajo tensiones desde el principio.

Es evidente que si en este caso se tiene todavía una dilatación del bizcocho por efecto de la humedad, fácilmente se pueden formar tensiones que pueden conducir a la formación de fisuras.

Sin embargo, en el esmalte al circonio viscoso la interfase es tan sutil que ésta no ejerce ninguna influencia apreciable. Puesto que en el sistema de tres estratos (bizcocho-interfase-esmalte) no pueden observarse enseguida los verdaderos síntomas de tensiones, el problema de un bizcocho con esmalte de plomo ha tenido que ser afrontado cuantitativamente.

\section{PARTE SEGUNDA}

\section{Cálculo de las tensiones}

H. J. OEL Y A. DIETZEL.

Si en el esmalte de plomo no se hubiese formado el estrato intermedio, la dilatación del 
esmalte, en un sistema plano de dos estratos, se calcularía (5) según la fórmula:

$$
\varepsilon_{\mathrm{g}}=-\frac{\left(\alpha_{\mathrm{s}}-\alpha_{\mathrm{g}}\right) \Delta t}{1+\frac{E_{\mathrm{g}}{ }^{*} \cdot d_{\mathrm{g}}}{E_{\mathrm{s}}{ }^{*} \cdot d_{\mathrm{s}}}}
$$

El valor $\left(1-\mu_{\mathrm{s}}\right)\left(1-\mu_{\mathrm{g}}\right)$, en el cual figuran las constantes de Poisson, se elimina del denominador. En la fórmula $\alpha$ es, debido a la similitud de $\mu_{\mathrm{s}}$ y $\mu_{\mathrm{g}}$, lineal entre ET y la temperatura ambiente, es decir, en la zona de $\Delta t$. Significando: $E^{*}$ Módulo $E, d$ espesor, $s$ bizcocho, $g$ esmalte.

Entonces, según la figura 1:

$$
\begin{aligned}
& \alpha_{\mathrm{s}} \cdot \Delta t=0,440 \times 10^{-2} \mathrm{~mm} / \mathrm{mm} ; \\
& \alpha_{\mathrm{g}} \cdot \Delta t=0,397 \times 10^{-2} \mathrm{~mm} / \mathrm{mm} ;
\end{aligned}
$$

además:

$$
\begin{aligned}
E_{\mathrm{g}}{ }^{*} & =6.400 \mathrm{kp} / \mathrm{mm}^{2} ; \\
d_{\mathrm{g}} & =0,28 \mathrm{~mm} \text { (ver fig. 6); } \\
E_{\mathrm{s}}{ }^{*} \text { húmedo } & =1.490 \mathrm{kp} / \mathrm{mm}^{2} ; \\
d_{\mathrm{s}} & =5 \mathrm{~mm} .
\end{aligned}
$$

En cuyo caso para la dilatación del esmalte se tendrá:

$$
\varepsilon_{\mathrm{g}}=-0,0347 \% \text {. }
$$

y para $\sigma=\frac{E^{*}}{1-\mu} \cdot \varepsilon$ se calculará una tensión de compresión de:

$$
\sigma_{\mathrm{g}}=2,96 \mathrm{kp} / \mathrm{mm}^{2} \text {. }
$$

Como ya habíamos deducido cualitativamente, la dilatación por humedad no bastará para compensar la compresión existente en el esmalte (que habíamos calculado someramente).

A tal efecto necesitamos observar la influencia del estrato intermedio.

En un azulejo plano, las dilataciones $\varepsilon$ de los tres estratos se calculan paralelamente a su superficie, según Oel, del siguiente modo (6):

$$
\begin{aligned}
& \varepsilon_{1}=\frac{E_{2} \cdot \alpha_{12}+E_{3} \cdot \alpha_{12}+E_{3} \cdot \alpha_{23}}{E_{1}+E_{2}+E_{3}} ; \\
& \varepsilon_{2}=\frac{E_{3} \cdot \alpha_{23}-E_{1} \cdot \alpha_{12}}{E_{1}+E_{2}+E_{3}} ; \\
& \varepsilon_{3}=\frac{E_{1} \cdot \alpha_{12}+E_{2} \cdot \alpha_{23}+E_{1} \cdot \alpha_{23}}{E_{1}+E_{2}+E_{3}} ;
\end{aligned}
$$

En las cuales:

$$
E=\frac{E^{*} \cdot d}{1-\mu}
$$

Siendo:

$$
\begin{aligned}
E^{*} & =\text { módulo de elasticidad. } \\
d & =\text { espesor de la capa. } \\
\mu & =\text { constante de Poisson. } \\
\alpha_{12} & =\alpha_{1}-\alpha_{2} . \\
\alpha_{23} & =\alpha_{2}-\alpha_{3} .
\end{aligned}
$$

$\alpha$ es la diferencia de longitud $\Delta l / l$ como consecuencia de la contracción térmica entre 
la temperatura de enfriamiento del esmalte y de los estratos intermedios (es decir, todavía en un estado en que las tensiones son nulas) y la temperatura ambiente. Como consecuencia de los cálculos y de las hipótesis se pueden establecer los siguientes valores:

- esmalte de plomo sin alterar (1):

$$
\begin{aligned}
E_{1}^{*} & =6.400 \mathrm{kp} / \mathrm{mm}^{2} \\
d_{1} & =0,20 \mathrm{~mm} ; \\
\mu_{1} & \approx 0,25 \\
E T_{1} & =576^{\circ} \mathrm{C} \\
\alpha_{1} & =0,397 \times 10^{-2} \mathrm{~mm} / \mathrm{mm} ;
\end{aligned}
$$

- estrato intermedio (2):

$$
\begin{aligned}
E_{2}{ }^{*} & \approx 7.000 \mathrm{kp} / \mathrm{mm}^{2} ; \\
d_{2} & =0,08 \mathrm{~mm} ; \\
\mu_{2} & \approx 0,25 ; \\
E T_{2} & =593^{\circ} \mathrm{C} ; \\
\alpha_{2} \text { hasta } 593^{\circ} \mathrm{C}\left(E T_{2}\right) & =0,378 \times 10^{-2} \mathrm{~mm} / \mathrm{mm} ; \\
\alpha_{2} \text { hasta } 576^{\circ} \mathrm{C}\left(E T_{1}\right) & =0,309 \times 10^{-2} \mathrm{~mm} / \mathrm{mm} ;
\end{aligned}
$$

- bizcocho húmedo (3):

$$
\begin{aligned}
E_{3}{ }^{*} & =1.490 \mathrm{kp} / \mathrm{mm}^{2} ; \\
d_{3} & =5 \mathrm{~mm} ; \\
\mu_{3} & =0,25 ; \\
\alpha_{3} \text { hasta } 593^{\circ} \mathrm{C} & =0,450 \times 10^{-2} \mathrm{~mm} / \mathrm{mm} .
\end{aligned}
$$

Por tanto:

$$
\begin{aligned}
& \alpha_{12}=+0,088 \times 10^{-2} \mathrm{~mm} / \mathrm{mm} ; \\
& \alpha_{23}=-0,072 \times 10^{-2} \mathrm{~mm} / \mathrm{mm} ;
\end{aligned}
$$

que permiten calcular las dilataciones:

$$
\begin{aligned}
& \varepsilon_{1}=+0,018 \% \text { (barniz de plomo) } \\
& \varepsilon_{2}=-0,070 \% \text { (estrato intermedio) } \\
& \varepsilon_{3}=+0,0021 \% \text { (bizcocho). }
\end{aligned}
$$

Siendo $\sigma=\frac{E^{*}}{1-\mu} \cdot e$, se pueden calcular también las tensiones.

La parte principal del barniz de plomo, debido a la capa intermedia, se encuentra después de la cocción bajo una tensión de tracción igual a $1,5 \mathrm{kp} / \mathrm{mm}^{2}$ y no bajo una solicitación de compresión, como sucedería si no existiese la capa intermedia. La dilatación por humedad se suma ahora a la dilatación que se tenía al final de la iniciación, y se eleva por lo tanto la solicitación a la tracción en el esmalte de plomo que permanece inalterado.

Esto es válido para el azulejo que se había observado con dificultad. Cuando se coloca en el muro este dato todavía varía algo. (Ver parte IV).

De esta manera se puede establecer cuantitativamente que la solicitación inicial de compresión que se presenta en el barniz de plomo se concentra exclusivamente en la capa 
intermedia, mientras que en el propio barniz comienza a presentarse una solicitación de tracción.

Se ha estado tratando de rebajar todavía más el coeficiente de dilatación de la frita, pero el resultado ha sido que el azulejo se abarquilla cuando se saca del horno.

Sin embargo, aunque las solicitaciones de compresión que llegaban a alcanzarse no abarcaron a toda la sección horizontal del barniz de plomo, cabe atribuir la causa del abarquillamiento únicamente a la capa intermedia, puesto que en el presente caso ésta se encontraba ya bajo una solicitación de compresión de $6 \mathrm{kp} / \mathrm{mm}^{2}$.

Ahora ya es posible comprender, además, por qué la prueba Steger no aportaba ninguna aclaración. En este caso la tensión de compresión se producía en el estrato intermedio y no en el esmalte.

\section{PARTE TERCERA}

\section{Medidas efectuadas sobre el mortero y sobre las paredes}

\section{A. DIETZEL.}

\section{- Muros de ensayo}

Los ensayos con azulejos fijados a la pared se han realizado sobre dos tipos de muros diferentes. En un caso sobre un muro de Ytong (de $2 \mathrm{~m}$ de altura, $1 \mathrm{~m}$ de longitud y $10 \mathrm{~cm}$ de espesor), y que se encuentra sentado sobre una base de cemento (fig. 8). Se puso en evidencia que los muros de Ytong cambian, de una forma reversible, su masa en relación con el contenido de agua. En vista de ello se puso a punto un nuevo campo de medidas en la parte central de un muro de ladrillo, que tenía cerca de 80 años y que se encontraba a la intemperie (fig. 9). En este muro los cambios dimensionales, debidos a va-
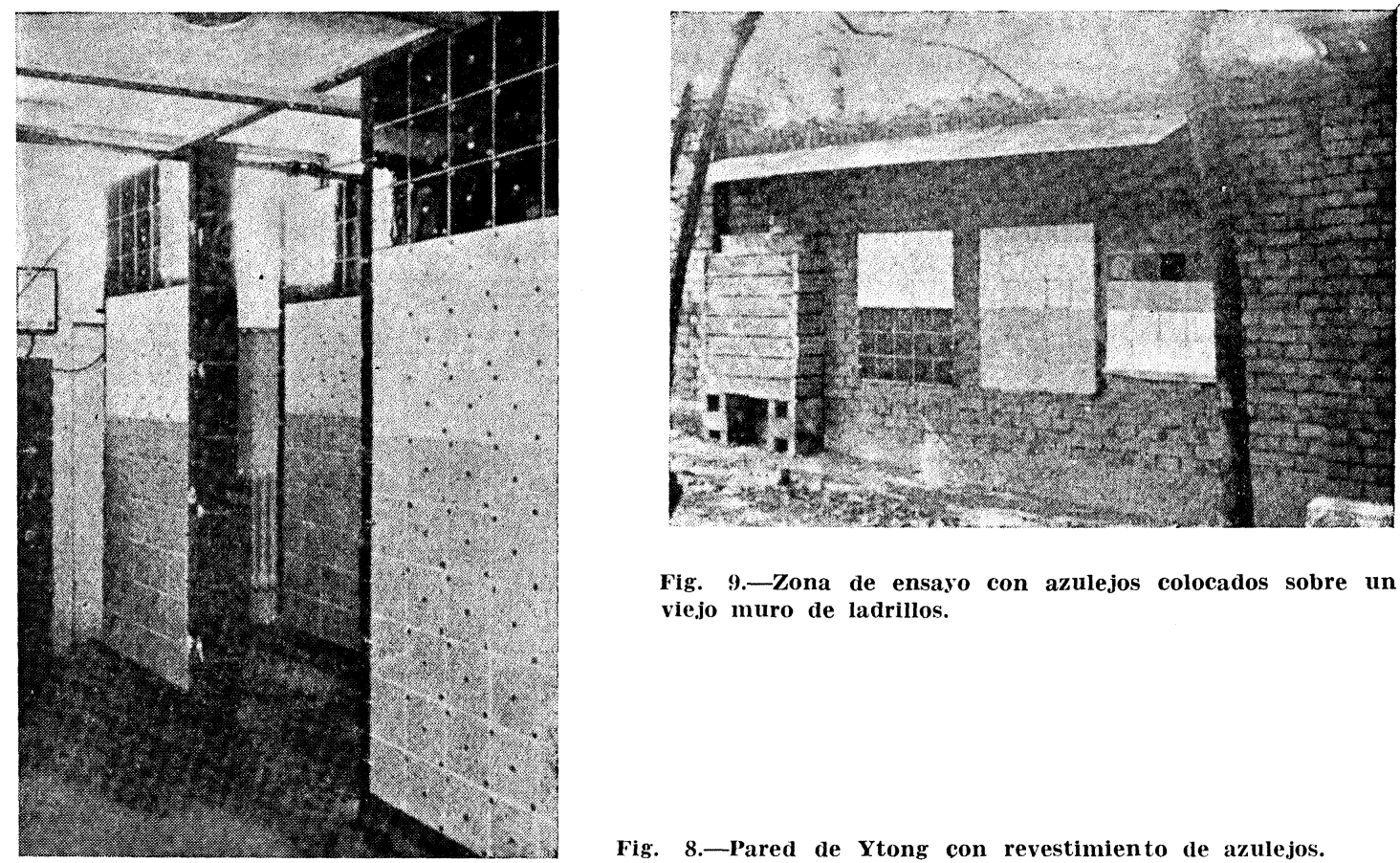

Fig. 9.-Zona de ensayo con azulejos colocados sobre un vie.jo muro de ladrillos.

Fig. 8.-Pared de Ytong con revestimiento de azulejos. 
riaciones de la temperatura, eran mucho más apreciables que los obtenidos en el muro de Ytong; en consecuencia, en este segundo caso se podían seguir mejor los cambios dimensionales del azulejo.

\section{Mortero}

Para la preparación de los morteros se utilizó cemento portland PZ 275 junto con cuatro tipos de arena. La dosificación fue de una parte de cemento y cinco partes de arena (en volumen).

Estas diferentes arenas procedían de Hamburgo, Bonn, Augsburg y Würzburg. En la tabla III pueden verse sus granulometrías.

T A B L A III

Granulometrías de las distintas arenas

\begin{tabular}{|c|c|c|c|c|}
\hline$(\mathrm{mm})$ & $\begin{array}{c}\text { Hamburgo } \\
(\% \text { en peso })\end{array}$ & $\begin{array}{c}\text { Bonn } \\
(\% \text { en peso })\end{array}$ & $\begin{array}{c}\text { Augsburg } \\
(\% \text { en peso })\end{array}$ & $\begin{array}{c}\text { Würzburg } \\
(\% \text { en peso })\end{array}$ \\
\hline 0 & $-0,060$ & - & 4,36 & 11,76 \\
$0,060-0,10$ & 0,01 & 0,80 & 1,94 & 0,01 \\
$0,010-0,2$ & 0,13 & 1,88 & 8,41 & 0,68 \\
$0,2-0,3$ & 3,10 & 4,36 & 10,50 & 2,43 \\
$0,3-0,4$ & 24,75 & 12,34 & 10,50 & 6,21 \\
$0,4-0,5$ & 21,74 & 11,44 & 8,11 & 16,80 \\
$0,5-1,0$ & 45,12 & 48,10 & 9,74 & 11,81 \\
$>1,0$ & 5,15 & 16,72 & 39,04 & 46,91 \\
\end{tabular}

T A в L A IV

Composición del mortero

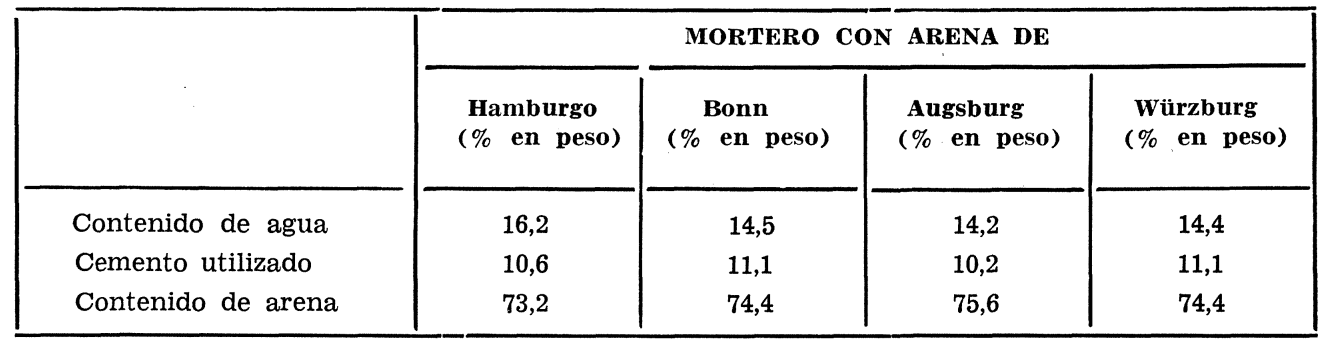

Para conseguir que estos experimentos correspondieran con lo que normalmente sucede en la práctica se le confió una amplia libertad al solador en lo que se refiere a la adición de agua. Los morteros ya listos para su utilización, después de haber sido bien amasados, dieron las composiciones que se indican en la tabla IV. El mortero más difícil de trabajar fue el que se hizo con arena de Hamburgo: la arena comenzó a separarse cuando se secó, y una vez colocada la loseta resbaló al tratar de fijarla en la pared. Es sorprendente el hecho de que la arena de Hamburgo tenga más de un $95 \%$ de granos con tamaños comprendidos entre 0,3 y $1,0 \mathrm{~mm}$.

En el mortero se determinaron diversas propiedades físicas, siendo la más interesante de todas la retracción, que es la representada en la figura 10.

\section{Colocación de las losetas}

Antes de su fijación, el solador sumerge durante unos segundos, de vez en cuando, gru- 
pos de 8-10 azulejos. Con esto se trata de establecer cualquier influencia, debida a diferencias de absorción de agua, que pueda tener sobre la capacidad y poder de adherencia.

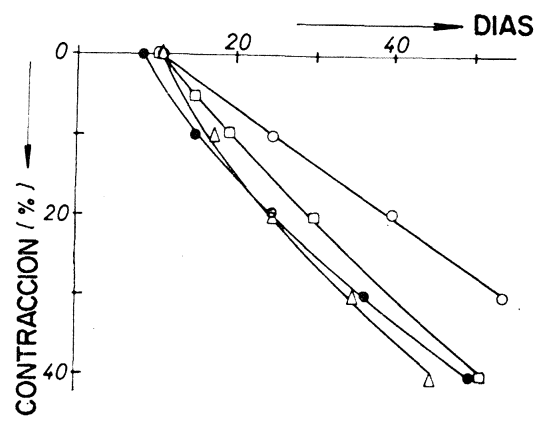

Fig. 10.-Curvas de retracción de diferentes morteros con arenas de diversas procedencias: 0 Arena de Bonn, $\square$ Arena de Augsburg, - Arena de Hamburgo, $\Delta$ Arena de Würzburg.

Las losetas secas absorben gran parte del agua de la capa de mortero de agarre, por lo cual se desprenden fácilmente y se caen.

Por otra parte si se saturan de agua los azulejos éstos no agarran y se resbalan todavía más. Como puede verse en la tabla $\mathrm{V}$ el contenido de agua que ha de tener ei azulejo para que se adhiera a la capa vertical de mortero es relativamente pequeño. Los contenidos de agua son los que obtiene el solador al sumergir durante unos segundos los azulejos.

T A B L A V

Contenido de agua del azulejo al colocarlo

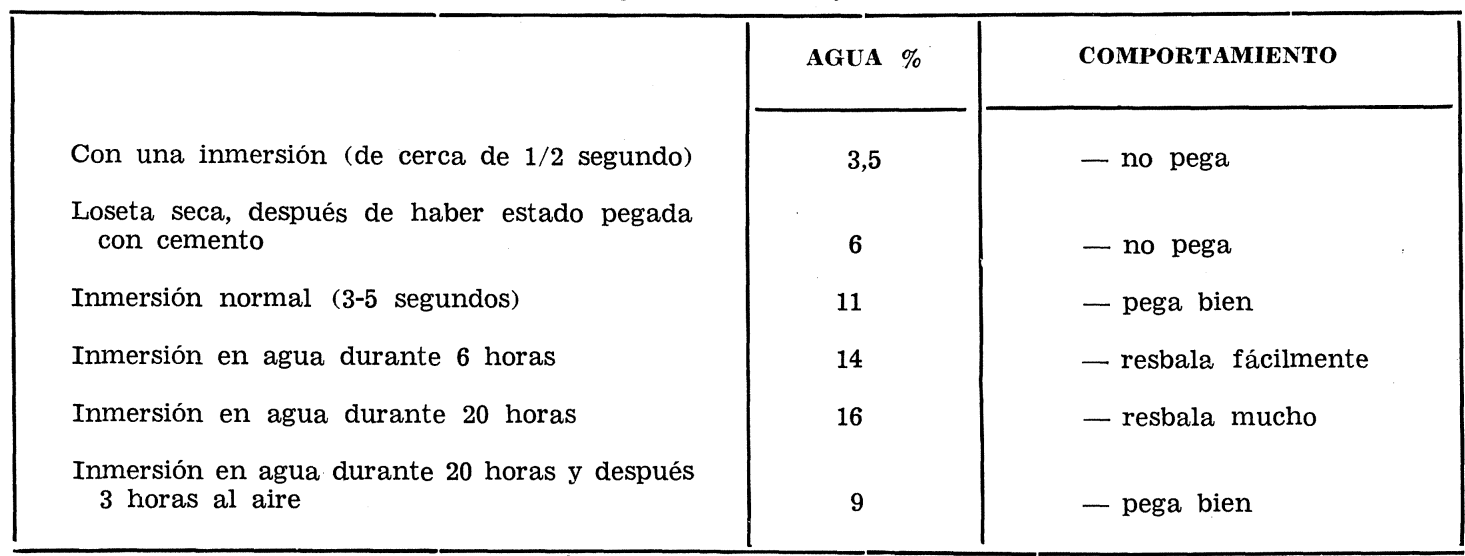

\section{Mortero de agarre}

El mortero utilizado para el agarre esta hecho a base de cemento blanco corriente, y en los casos necesarios, con una adición de cuarzo (o cal) en la proporción de 1:1. También se determinó el valor del módulo $E$ pero, debido al pequeño espesor del mortero de cemento de agarre, éste resultó de importancia nula.

\section{PARTE CUARTA}

\section{Examen de los azulejos después de su colocación}

A. DIETZEL.

\section{Capacidad de adherencia del azulejo al mortero}

En las plaquetas esmaltadas, no fijadas, la dilatación por humedad sigue una curva tal 
que la cara esmaltada se encuentra en la cara cóncava del azulejo. La figura 11 muestra la curvatura de un azulejo en libertad de movimientos, realizado sobre tiras talladas y al cabo de 50 días. Cabe pensar que, como consecuencia de esta deformación del azulejo, se desprenda.

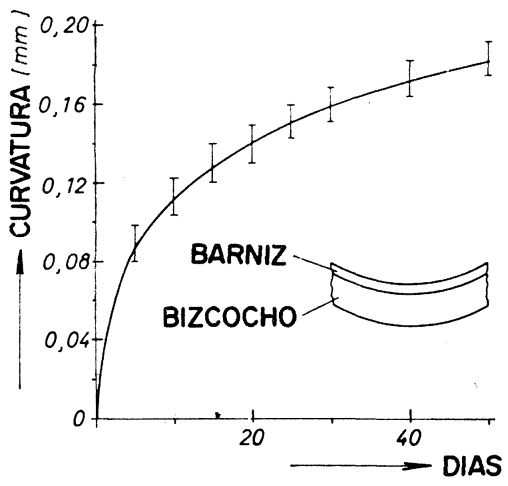

Fig. 11.-Curvatura del azulejo cuando éste se encuentra en libertad de movimientos.

A tal fin se utilizó el dispositivo que puede verse en la figura 12.

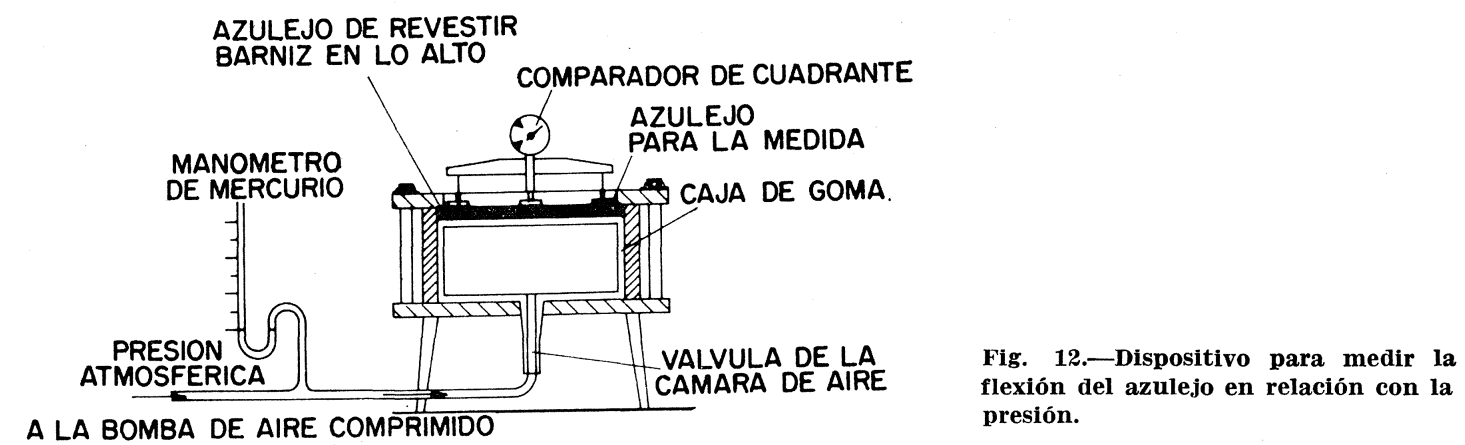

Para la flexión alcanzada al cabo de 50 días fue necesaria una presión de $0,2 \mathrm{kp} / \mathrm{mm}^{2}$, como término medio.

Con el dispositivo que se indica en la figura 13 se ha tratado de despegar un azulejo de la pared de ensayos, que estaba fijado desde hacía 2 años.

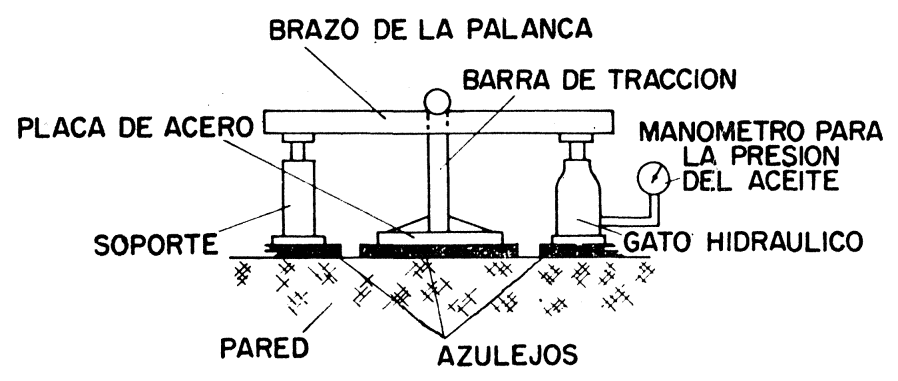

Fig. 13.-Dispositivo para medir la capacidad de adhesión del azulejo después de colocado.

Para este experimento fue necesaria una fuerza mayor que la que se podía obtener con el dispositivo precedente, y que ha sido preciso diseñar así, puesto que el anterior, que era un simple brazo de palanca, resultó insuficiente. Para los diversos morteros utilizados se obtuvieron los valores de adherencia que figuran en la tabla VI. 
T A B L A VI

Valores de la medida de la fuerza adhesiva entre el azulejo y el mortero

\begin{tabular}{|c|c|c|}
\hline Mortero con Arena de: & $\begin{array}{c}\text { Adherencia del azulejo } \\
\left(\mathrm{kp} / \mathrm{cm}^{2}\right)\end{array}$ & $\begin{array}{l}\text { Marcha del ensayo } \\
\text { al arrancamiento: }\end{array}$ \\
\hline Augsburg & $>4,1$ & $-\sin$ rotura \\
\hline Hamburgo & $>3,7$ & $-\sin$ rotura \\
\hline Bonn & $>4,0$ & $-\sin$ rotura \\
\hline Würzburg & 3,1 & - rotura del mortero \\
\hline
\end{tabular}

Es preciso hacer constar que, según las conclusiones de los experimentos a largo plazo realizados durante 2 años, no se consiguió despegar ningún azulejo sin dañarlo. Se ha llegado a comprobar que en los azulejos fijados según la norma, la fuerza adhesiva era aproximadamente un múltiplo del valor de la fuerza que se determina en la dilatación por humedad y que tiende a curvar al azulejo. Cuando el azulejo se fija en la pared y sucede lo contrario, significa que éste no ha sido fijado según las normas.

\section{Variaciones longitudinales del azulejo después de su colocación y su curvatura}

Las variaciones longitudinales del azulejo, después de la fijación, se han medido sobre tres azulejos (*) dispuestos de la forma que se indica en la figura 14.

Para hacer las medidas de cambios longitudinales con el comparador de reloj se utilizó un tubo de cristal de cuarzo.

Para medir la curvatura se empleó el dispositivo que se muestra en la figura 15.

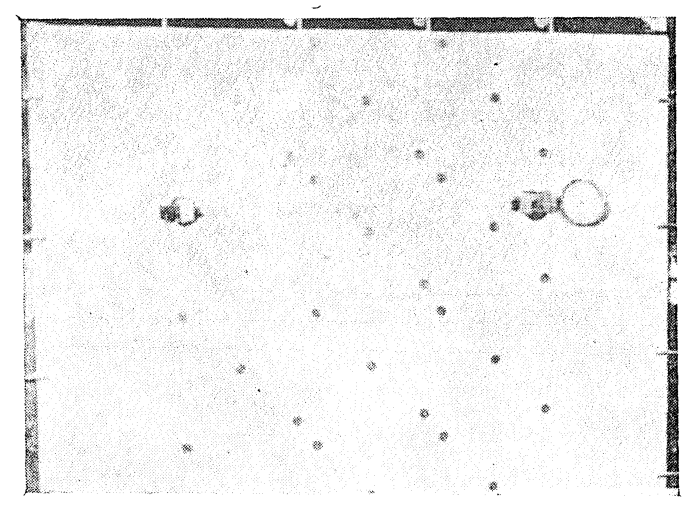

Fig. 14.-Medida de la variación de longitud de los azulejos colocados efectuada con comparador de reloj. No se han empleado los puntos de medida que se observan en los extremos derecho e izquierdo.

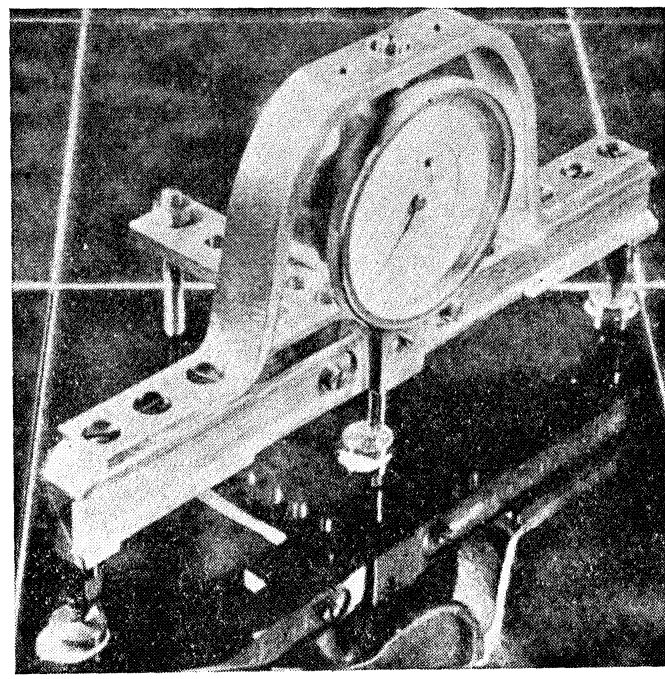

Fig. 15. - Esquema para la medida de la curvatura del azulejo después de colocado.

(*) En los tabiques dispuestos para el ensayo se dispuso una fila con cinco azulejos. Al principio, los puntos para la determinación de las variaciones longitudinales de los cinco azulejos se fijaron sobre los dos más extremos, con objeto de poder determinar las variaciones longitudinales totales. Esto, sin embargo, implicó errores de medida, a causa de la retracción del mortero, pues los azulejos externos se achaflanaron algo. 
Para poder garantizar que los comparadores de reloj se colocaron en el punto exacto se pegaron en los azulejos, con cemento, tres plaquitas de latón, y se anotaron los valores iniciales de la tensión que provocaba la curvatura.

Pronto pudo observarse que los valores de las medidas sobre los azulejos de las paredes de Ytong no estaban influenciadas decisivamente por las variaciones de temperatura, sino que su mayor influencia se debía, en primer lugar, a la cantidad de humedad que contenía la propia pared.

Para probar esto se roció la pared, de vez en cuando, con agua, con objeto de obtener - una dilatación, por humedad del bizcocho, que fuese la mayor posible.

Los valores obtenidos sobre el viejo muro de ladrillo (fig."16) estuvieron más próximos a lo que era de esperar.

En la curva que indica las variaciones longitudinales se pueden apreciar dos partes diferentes: para los ocho primeros días la dilatación de un azulejo fijado es cerca del doble que la de un azulejo en libertad de movimientos (fig. 2); en estas circunstancias el mortero es todavía muy flexible. Sin embargo, después la dilatación es mucho más lenta y sustancialmente corresponde con la marcha de las temperaturas del muro a la intemperie (de aquí las oscilaciones de los valores de las medidas).

Otro fenómeno muy importante es la medida de la curvatura del azulejo en el transcurso del tiempo. Al contrario de lo que le sucede a la plaqueta libre, la plaqueta que está fijada se curva a todo lo largo de la parte del bizcocho (fig. 17).

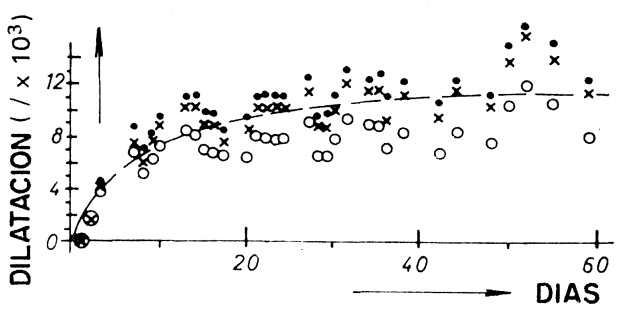

Fig. 16.-Valores de la dilatación, dentro de una zona de medidas, en un muro de ladrillo. - Fila superior; $X$ fila media; 0 fila inferior.

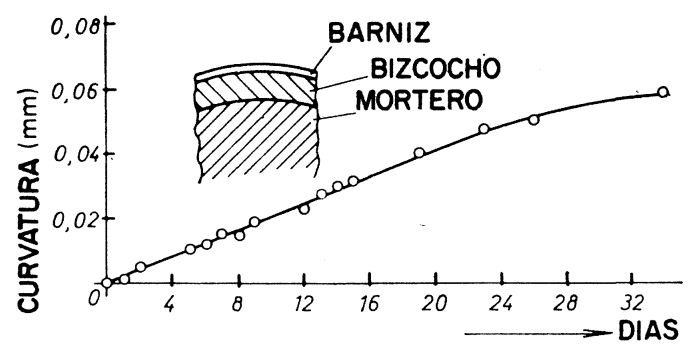

Fig. 17.-Forma de la curvatura de un azulejo después de su colocación, que se produce hacia la parte del bizcocho (contrariamente a lo que sucede en la figura 11).

Este fenómeno es causado por el mortero al retraerse durante el fraguado (fig. 10).

Esta observación tiene una enorme importancia desde el punto de vista del experimento técnico. Generalmente, el examen de la dilatación por humedad se efectúa sobre plaquetas sueltas sin fijar. Pero esta forma de hacerlo da lugar a la posibilidad de que se absorban una parte de las tensiones que se desarrollan. Por el contrario, en las plaquetas que se encuentran fijadas no cabe esta posibilidad y, en consecuencia, vienen obligadas a curvarse por la acción del mortero, de tal modo que el esmalte se encuentra sometido a una dilatación adicional (fig. 17).

Es decir, los azulejos esmaltados, una vez afectados por la humedad, se dejarán en este estado durante algunas horas antes de proceder a valorar su tendencia a la formación de fisuras capilares.

En estas condiciones y en el sistema de tres capas: azulejo-mortero-pared, se pueden cal- 
cular cuáles son las dilataciones (o las compresiones) generales en la plaqueta, que se producen al actuar la dilatación por humedad o por la retracción del mortero de agarre.

Además es preciso tener en cuenta que una parte de la dilatación por humedad se produce en un momento en el que el mortero se halla todavía fresco.

En la ecuación dạda anteriormente (parte segunda) se pueden incluir los siguientes valores:

Azulejo bañado (1):

$$
\begin{aligned}
E_{1}^{*} & \approx 1.490 \mathrm{kp} / \mathrm{cm}^{2} ; \\
d_{1} & =5 \mathrm{~mm} \\
\mu_{1} & \approx 0,25 \\
\alpha_{1} & =-0,6 \times 10^{-4} \mathrm{~mm} / \mathrm{mm} ;
\end{aligned}
$$

(dilatación por humedad desde 7 días hasta 50 días).

Mortero (2):

$$
\begin{aligned}
E_{2}{ }^{*} & \approx 1.000 \mathrm{kp} / \mathrm{cm}^{2} ; \\
d_{2} & =10 \mathrm{~mm} ; \\
\mu_{2} & \approx 0,25 \\
\alpha_{2} & =+4 \times 10^{-4} \mathrm{~mm} / \mathrm{mm} ;
\end{aligned}
$$

(retracción al cabo de 50 días).

Muro (3):

$$
\begin{aligned}
{E_{3}}^{*} & \approx 1.000 \mathrm{kp} / \mathrm{cm}^{2}(7) ; \\
d_{3} & =120 \mathrm{~mm} ; \\
\mu_{3} & \approx 0,25 \\
\alpha_{3} & =0 .
\end{aligned}
$$

Con lo cual se produce una torsión en el azulejo del orden de un 0,0086 \% y una solicitación de compresión de $0,17 \mathrm{kp} / \mathrm{mm}^{2}$.

En conclusión, esta cifra nos indica que si el azulejo se coloca con cuidado no se producirán daños.

En lo que se refiere al esmalte es necesario considarar que, como consecuencia del curvado (no provocado por el esmalte) (fig. 17) se produce en el mismo una dilatación del $0,0063 \%$. Por eso no todo el encurvamiento de la plaqueta está relacionado con el esmalte $(0,0023 \%)$. La dilatación que se produce en el esmalte después de la cocción (aparte de la dilatación por humedad del bizcocho), y que es del 0,018 \% (véase parte segunda) a consecuencia de la colocación, no tiene casi efecto alguno, alcanzando valores del 0,016\%.

\section{Otras influencias}

Ya se ha dicho anteriormente que en el caso del barniz de plomo existen diversos factores que influyen en la formación de fisuraciones, de los cuales, ciertamente, el más importante es la formación de la capa intermedia, ya que ésta da lugar a la formación de tensiones al principio. Cuando el esmalte se encuentra bajo una ligera tensión no se producen, necesariamente, fisuraciones, puesto que la capacidad resistente de los esmaltes de 
plomo está comprendida entre 4 y $6 \mathrm{kp} / \mathrm{mm}^{2}$, y, según los cálculos, el esmalte se encuentra dentro de una dilatación del $0,18 \%$ (o respectivamente del $0,016 \%$ ), habiéndose establecido que la tensión de rotura deberá ser del $0,67 \%$.

Ahora bien, desde el punto de vista químico, los barnices de plomo no son muy resistentes. La superficie, donde generalmente se genera una fisuración, se encuentra de hecho "debilitada" por el agua utilizada para su fijación y por efecto de la acción de la humedad atmosférica.

Además de todo esto sucede que en la superficie del azulejo, debido al transporte, del baño de agua previo a la colocación, de la acción de la paleta durante el asiento y del lavado con esponja una vez colocados, se forman innumerables fisuras microscópicas y arañazos.

En consecuencia es fácil que cualquier pequeña dilatación, formada en el bizcocho por la acción de la humedad, pueda dar lugar a la formación de fisuras capilares.

\section{N O T A}

(1) La temperatura de "templado" ET es aquélla en la cual (al descender la temperatura) la curva de dilatación empieza a apartarse de su recorrido, que hasta entonces era rectilíneo. Esto es un síntoma que nos indica que del estado pastoso se está pasando a un estado de fragilidad. ET se encuentra un poco por encima de la temperatura de transformación "tg". Tanto ET como tg dependen de la velocidad de calentamiento o enfriamiento. En el presente caso se ha observado por el dilatómetro, que la temperatura de calentamiento es comparable a la velocidad de enfriamiento en el horno cerámico.

(2) H. G. Schurecht: J. Amer. Ceram. Soc. 11 (1928), 271 W. Finke: Silikattechnik 20 (1969), 257.

(3) Véase nota (4).

(4) H. Scholze, J. Müller y U. Hildebrandt: Ber. Dt. Keram. Ges. 47 (1970), 45-49.

(5) Véase trabajo de G. Matz y P. Gayer: Berechung von gusseisernen emallierten Druckbehälters. (Página 162); edición Springer-Verlag Berlín-Göttingen-Heidelberg. 1959, e (1).

(6) H. G. Oel; VDI-Z. 108, 1727, 1966.

(7) Valores probables; véase publicación: Ziegeltaschenbuch de 1959, pág. 19. O también (en lo que se refiere a losetas de solar): A. Dietzel y M. Weisner-Kieffer: Ber. Dt. Keram. Ges. 30 275, 1953. 